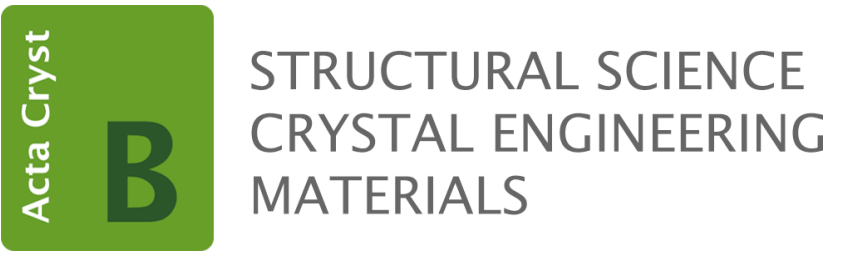

Volume 76 (2020)

Supporting information for article:

The effective volumes of waters of crystallization: general organic solids

Leslie Glasser 
$\underline{\text { Supplementary Information }}$

\title{
The Effective Volumes of Waters of Crystallization: General Organic Solids
}

\author{
Leslie Glasser \\ Curtin Institute for Computation, Discipline of Chemistry \\ Curtin University, GPO Box U1987, Perth, WA 6845, Australia
}

3 Figures $\sim 2000$ words

Supplementary Tables S1 and S2, Figure S1

${ }^{*}$,a Corresponding author

L. Glasser: $\quad$ Telephone: +618 9848-3334

E-mail: 1.glasser@curtin.edu.au

ORCID iD: 0000-0002-8883-0564 
Table S1: Asymmetric-unit volumes, $V_{\mathrm{m}} / \AA^{3}$, as a function of temperature, $T / \mathrm{K}$, for the anhydrates (CSD refcodes PUBMUUmn) and hydrate (CSD refcodes PUBMIImn) of hexanitro-azaisowurtzitane (HNIW, $\mathrm{C}_{6} \mathrm{H}_{6} \mathrm{~N}_{12} \mathrm{O}_{12}$ ). The data is sorted by temperature, and then by asymmetric-unit volume.

\begin{tabular}{|c|c|c|c|}
\hline $\mathrm{C}_{6} \mathrm{H}_{6} \mathrm{~N}_{12} \mathrm{O}_{12}$ & $V_{m} / \AA^{3}$ & $T / K$ & Polymorph \\
\hline Anhydrate & & & $\mathrm{Pb} 2_{1} \mathrm{a}$ \\
\hline PUBMUU24 & 365.981 & 0 (?) & $\beta$ \\
\hline PUBMUU03 & 366.435 & 298 & $\beta$ \\
\hline \multirow[t]{2}{*}{ PUBMUU01 } & 366.495 & 298 & $\beta$ \\
\hline & & & $\mathrm{P} 2{ }_{1} / \mathrm{n}$ \\
\hline PUBMUU25 & 348.829 & 100 & $(\varepsilon)$ \\
\hline PUBMUU12 & 349.312 & 100 & $\varepsilon$ \\
\hline PUBMUU13 & 350.420 & 150 & $\varepsilon$ \\
\hline PUBMUU14 & 353.001 & 200 & $\varepsilon$ \\
\hline PUBMUU15 & 355.010 & 250 & $\varepsilon$ \\
\hline PUBMUU20 & 352.708 & 298 & $\varepsilon$ \\
\hline PUBMUU02 & 356.036 & 298 & $\varepsilon$ \\
\hline PUBMUU17 & 356.742 & 298 & $\varepsilon$ \\
\hline PUBMUU19 & 356.781 & 298 & $\varepsilon$ \\
\hline PUBMUU21 & 357.122 & 298 & $\varepsilon$ \\
\hline PUBMUU18 & 357.217 & 298 & $\varepsilon$ \\
\hline PUBMUU16 & 357.559 & 298 & $\varepsilon$ \\
\hline PUBMUU05 & 358.005 & 298 & $\varepsilon$ \\
\hline \multirow[t]{2}{*}{ PUBMUU20a } & 356.326 & 383 & $\varepsilon$ \\
\hline & & & $\mathrm{P} 2_{1} / \mathrm{n}$ \\
\hline PUBMUU07 & 372.488 & 100 & $\gamma$ \\
\hline PUBMUU08 & 375.695 & 150 & $\gamma$ \\
\hline PUBMUU09 & 375.437 & 200 & $\gamma$ \\
\hline PUBMUU10 & 377.336 & 250 & $\gamma$ \\
\hline PUBMUU04 & 377.825 & 298 & $\gamma$ \\
\hline PUBMUU06 & 379.352 & 298 & $\gamma$ \\
\hline PUBMUU & 379.721 & 298 & $\gamma$ \\
\hline \multirow[t]{2}{*}{ PUBMUU11 } & 379.997 & 298 & $\gamma$ \\
\hline & & & $\mathrm{P} 2_{1} / \mathrm{n}$ \\
\hline PUBMUU23 & 319.774 & 298 & $\zeta$ \\
\hline Hydrate $0.25 \mathrm{H}_{2} \mathrm{O}$ & & & Pbca \\
\hline PUBMII02 & 363.889 & 86 & $\alpha$ \\
\hline PUBMII & 371.190 & 298 & $\alpha$ \\
\hline PUBMII01 & 373.159 & 298 & $\alpha$ \\
\hline
\end{tabular}


Table S2: Asymmetric-unit volumes, $V_{\mathrm{m}} / \AA^{3}$, of hydrate and anhydrate pairs with the temperatures of their determination, number of waters of crystallization per hydrate, $n\left(\mathrm{H}_{2} \mathrm{O}\right)$, and effective volume per water of crystallization corrected to $298 \mathrm{~K}, \mathrm{~V}_{\mathrm{m}}\left(\mathrm{H}_{2} \mathrm{O}\right)$, using a generic volumetric coefficient of thermal expansion of $147 \times 10^{-6} \mathrm{~K}^{-1}$. The CSD Refcodes appear in the final two columns. The data is sorted by the values of $V_{m}\left(\mathrm{H}_{2} \mathrm{O}\right)$, smallest to largest, with the most egregious outlier values listed in red italics. A value for t-butanol have been added to those from the source list. ${ }^{1}$

\begin{tabular}{|c|c|c|c|c|c|c|c|}
\hline$V_{\mathrm{m}}$ (hydrate) & $\mathrm{T}_{\text {hydr }} / \mathrm{K}$ & $n\left(\mathrm{H}_{2} \mathrm{O}\right)$ & $V_{m}(a n)$ & $\mathrm{T}_{\text {anhydr }} / \mathrm{K}$ & $V_{m}\left(\mathrm{H}_{2} \mathrm{O}\right)$ & Hydrate & Anhydrate \\
\hline 461.353 & 298 & 0.23 & 460.896 & 105 & -54.87 & LEZKAC & FIQFER \\
\hline 638.913 & 233 & 1 & 664.662 & 233 & -26.00 & PEGVEC & PEGVAY \\
\hline 823.370 & 173 & 0.5 & 842.710 & 298 & -8.42 & RAVBUL & NAFMEM \\
\hline 554.318 & 298 & 0.32 & 556.444 & 298 & -6.64 & BUJJIZ & DADMOK \\
\hline 655.425 & 298 & 2 & 663.342 & 250 & -6.30 & MAXVIQ & VIQJEK \\
\hline 475.978 & 298 & 1 & 480.125 & 298 & -4.15 & SURRIG & SURREC \\
\hline 465.466 & 298 & 1 & 469.446 & 298 & -3.98 & QOBJEW & QOBJAS \\
\hline 528.420 & 298 & 1 & 530.227 & 298 & -1.81 & VAXDUT & DEMYEZO1 \\
\hline 266.995 & 298 & 3 & 249.316 & 150 & 4.08 & MEPYRZ & MPYRAZ02 \\
\hline 438.804 & 298 & 0.5 & 436.579 & 298 & 4.45 & HEPNAR & HEPMUK \\
\hline 477.198 & 298 & 1 & 472.046 & 298 & 5.15 & AMEQAK & AMEPOX \\
\hline 597.687 & 298 & 2 & 585.637 & 298 & 6.03 & HEVKOI & ZECRUU \\
\hline 254.983 & 298 & 1 & 243.920 & 173 & 6.58 & SUNGUD & SUNGOX \\
\hline 233.112 & 298 & 1 & 226.450 & 298 & 6.66 & BOPQAY & COFDUW10 \\
\hline 658.867 & 298 & 1 & 652.043 & 298 & 6.82 & GAJMIN & GAJMOT \\
\hline 402.002 & 298 & 0.5 & 398.390 & 298 & 7.22 & PANYLB & ANPYAB \\
\hline 282.256 & 110 & 1 & 282.785 & 298 & 7.27 & IJESIZ & IJESAR \\
\hline 235.547 & 298 & 0.67 & 229.780 & 298 & 8.61 & BOCNIQ & TICYOT \\
\hline 258.228 & 298 & 2 & 240.786 & 298 & 8.72 & FONHEW & BTCOAC \\
\hline 274.149 & 298 & 1 & 259.698 & 173 & 9.68 & PHOLCL & CUZDIK \\
\hline 300.918 & 298 & 1 & 290.447 & 298 & 10.47 & THMPIM10 & IMOXSF \\
\hline 266.877 & 298 & 1 & 252.334 & 220 & 11.65 & QIHSIJ & CAWKEQ \\
\hline 512.151 & 298 & 0.25 & 509.148 & 298 & 12.01 & YILKUZ & SEBROG \\
\hline 260.964 & 298 & 0.5 & 254.320 & 298 & 13.29 & VEBREZ & GEYRAD \\
\hline 576.815 & 298 & 4 & 522.751 & 298 & 13.52 & JATDUD & JATFAL \\
\hline 485.680 & 298 & 1 & 472.016 & 298 & 13.66 & EVODOI & EVODUO \\
\hline 385.307 & 298 & 2 & 357.161 & 298 & 14.07 & CYSTIN10 & CYSTBR01 \\
\hline 380.782 & 298 & 0.5 & 363.017 & 100 & 14.40 & JEDTOB & TPEPHO03 \\
\hline 563.769 & 298 & 1 & 549.009 & 298 & 14.76 & DEPDEH & DEPDAD \\
\hline 359.269 & 298 & 1.5 & 336.464 & 298 & 15.20 & FIXPEH & BODSES \\
\hline 281.055 & 298 & 1 & 265.849 & 298 & 15.21 & VAMCAO & VAMBUH \\
\hline 690.726 & 298 & 1 & 675.383 & 298 & 15.34 & BUDTUP & BUDTOJ \\
\hline 250.914 & 298 & 1 & 235.391 & 298 & 15.52 & HECMUX & VUNYUY10 \\
\hline
\end{tabular}




\begin{tabular}{|c|c|c|c|c|c|c|c|}
\hline 349.691 & 150 & 2 & 318.937 & 150 & 15.71 & RALDEN01 & XEHKOK \\
\hline 119.361 & 298 & 0.5 & 111.332 & 298 & 16.06 & AHOXLH & MOYHAJ \\
\hline 300.285 & 298 & 1 & 284.188 & 298 & 16.10 & PHBARM & PHBARB06 \\
\hline 160.975 & 298 & 1 & 144.344 & 298 & 16.63 & NURAMH & NIMFOE \\
\hline 231.072 & 113 & 0.5 & 222.030 & 86 & 16.81 & TRMHXD & DMHXDM \\
\hline 422.832 & 205 & 2 & 389.264 & 205 & 17.01 & WAFPAV & WAFNUN \\
\hline 268.111 & 298 & 0.5 & 259.546 & 298 & 17.13 & BAJGUO & NASQAZ \\
\hline 420.262 & 298 & 1 & 400.886 & 263 & 17.31 & THIAMC12 & UNEXOA \\
\hline 177.728 & 298 & 0.5 & 166.767 & 205 & 17.36 & ZZZSBA01 & HUYBUY \\
\hline 659.486 & 298 & 1 & 642.002 & 298 & 17.48 & HAJWAR & HAJWIZ \\
\hline 173.797 & 298 & 1.5 & 142.966 & 82 & 17.53 & POTPET & GLYGLYO4 \\
\hline 594.912 & 298 & 0.16 & 592.072 & 298 & 17.75 & DOBLOV & CUVHAC01 \\
\hline 222.377 & 298 & 0.5 & 213.432 & 298 & 17.89 & QUINCX & QUINCB10 \\
\hline 424.741 & 150 & 2 & 389.159 & 150 & 18.18 & BAFDUI & BAFFAQ \\
\hline 685.917 & 298 & 1 & 667.578 & 298 & 18.34 & MEYRAJ & MEYQUC \\
\hline 640.270 & 298 & 1 & 621.922 & 298 & 18.35 & QAMCEN & QAMCAJ \\
\hline 212.032 & 95 & 4 & 142.724 & 298 & 18.91 & TFMSTH & TFMSUL02 \\
\hline 499.114 & 298 & 1 & 480.134 & 298 & 18.98 & CIZWEN & DAMVIX \\
\hline 371.190 & 298 & 0.25 & 366.435 & 298 & 19.02 & PUBMII & PUBMUU03 \\
\hline 262.519 & 298 & 1 & 243.208 & 298 & 19.31 & ARGHCL10 & LARGIN02 \\
\hline 466.700 & 298 & 2 & 421.175 & 193 & 19.51 & FELCIJ & FELCOP \\
\hline 653.262 & 298 & 1 & 633.545 & 298 & 19.72 & HOXGEG & HOXGIK \\
\hline 277.310 & 150 & 1 & 257.770 & 150 & 19.97 & BAFFIY & BAFFOE \\
\hline 202.000 & 123 & 2 & 167.140 & 298 & 20.03 & PYZDCX01 & IYAWAG \\
\hline 308.449 & 298 & 1 & 288.257 & 298 & 20.19 & BAFPAZ & CALDEY \\
\hline 337.336 & 298 & 1 & 317.104 & 298 & 20.23 & CIMGUA & CIMETD01 \\
\hline 265.613 & 298 & 1.5 & 235.159 & 298 & 20.30 & RIKBIW & RIKBOC \\
\hline 483.137 & 298 & 2 & 440.930 & 298 & 21.10 & TUTECD01 & THUTEC01 \\
\hline 433.661 & 298 & 2 & 391.293 & 298 & 21.18 & MITCDH01 & MITOMC \\
\hline 253.115 & 298 & 2 & 210.225 & 298 & 21.45 & DILFAF01 & FIFGOQ \\
\hline 306.850 & 233 & 1 & 285.541 & 236 & 21.64 & JEPJOD & BOBZAC02 \\
\hline 379.479 & 298 & 1 & 357.370 & 298 & 22.11 & BIMYEB & BIMYAX \\
\hline 165.710 & 298 & 2 & 117.865 & 100 & 22.21 & ZAHJIB & CYURAC12 \\
\hline 343.548 & 298 & 1 & 321.084 & 298 & 22.46 & TEJMIE & LETBER \\
\hline 323.492 & 263 & 1 & 302.678 & 298 & 22.48 & CETMPA01 & CLEOZP01 \\
\hline 617.697 & 253 & 4 & 528.587 & 263 & 22.62 & NURJEP01 & NURHOX \\
\hline 606.444 & 298 & 1 & 571.590 & 153 & 22.67 & CUWKAG & CUWJUZ \\
\hline 248.656 & 298 & 0.5 & 233.777 & 200 & 23.02 & YOYPIL & EGORIB \\
\hline 177.867 & 298 & 2 & 131.781 & 298 & 23.04 & BARBAD01 & BARBAC01 \\
\hline 366.670 & 298 & 1 & 337.287 & 173 & 23.19 & HILMEU & XOMMER \\
\hline 281.684 & 123 & 4 & 195.851 & 298 & 23.27 & NUYRAA & YAMSAG \\
\hline 190.903 & 298 & 1 & 164.625 & 178 & 23.37 & MSULIM & POMDAW01 \\
\hline 395.986 & 298 & 1 & 370.666 & 263 & 23.41 & FIMVOM & FESRIE \\
\hline 623.469 & 298 & 1 & 599.979 & 298 & 23.49 & UJOQUF & KAMPIY \\
\hline
\end{tabular}




\begin{tabular}{|c|c|c|c|c|c|c|c|}
\hline 430.384 & 298 & 1 & 406.874 & 298 & 23.51 & TESTOM & TESTON10 \\
\hline 215.337 & 298 & 1 & 191.480 & 298 & 23.86 & GLUCMH11 & GLUCSA10 \\
\hline 502.463 & 298 & 1 & 478.415 & 298 & 24.05 & QIJZUE & RORQEU01 \\
\hline 138.036 & 123 & 0.5 & 126.313 & 123 & 24.05 & RABBUN & NAGVUM \\
\hline 630.626 & 153 & 5 & 523.778 & 298 & 24.06 & BEQWID & LEWBOE \\
\hline 403.082 & 298 & 1 & 378.994 & 298 & 24.09 & ZZZTZQ01 & ZZZTSE01 \\
\hline 184.578 & 123 & 3 & 114.235 & 130 & 24.09 & GIXDIA & QATVUC \\
\hline 229.521 & 298 & 1 & 205.425 & 298 & 24.10 & WIKDAV & MUMBEL \\
\hline 138.545 & 298 & 1 & 110.869 & 100 & 24.45 & LSERMH10 & LSERIN20 \\
\hline 213.339 & 298 & 1 & 188.815 & 298 & 24.52 & SULAMH10 & SULAMD04 \\
\hline 433.921 & 298 & 1 & 409.255 & 298 & 24.67 & COVMEF & BARWUM01 \\
\hline 490.930 & 298 & 1.5 & 453.914 & 298 & 24.68 & QIDCIP & WIQMUE \\
\hline 327.372 & 298 & 1 & 302.502 & 298 & 24.87 & NOGKID & NOGKEZ \\
\hline 328.769 & 298 & 1 & 303.895 & 298 & 24.87 & PAKOJM & PAYKOJ \\
\hline 416.433 & 298 & 1 & 391.471 & 298 & 24.96 & SOHLAC & KOUMIN \\
\hline 267.457 & 298 & 4 & 164.458 & 173 & 24.99 & HOMPRO10 & QAMVAB \\
\hline 128.544 & 298 & 2 & 78.433 & 298 & 25.06 & OXACDH28 & OXALACO4 \\
\hline 222.282 & 298 & 0.25 & 215.995 & 298 & 25.15 & IBUXIN & UJEMEB \\
\hline 267.227 & 298 & 2 & 216.917 & 298 & 25.16 & HOSHEC & NANRAV \\
\hline 169.880 & 298 & 1 & 144.700 & 298 & 25.18 & VEXRUL & VEXREV \\
\hline 356.072 & 298 & 1.5 & 318.253 & 298 & 25.21 & FAYVUW & FAYVOQ \\
\hline 451.656 & 110 & 6 & 303.548 & 115 & 25.40 & PUHPOX & BOBHOP \\
\hline 138.947 & 123 & 2 & 89.329 & 130 & 25.49 & GIXDAS & ETDIAM01 \\
\hline 188.397 & 223 & 3 & 111.514 & 153 & 25.53 & PYRTHA10 & PYRDNA01 \\
\hline 164.298 & 298 & 1 & 138.742 & 298 & 25.56 & TARTDL01 & ZZZDUI01 \\
\hline 300.262 & 298 & 2 & 249.117 & 298 & 25.57 & PYMDSD & PYMSUL10 \\
\hline 178.544 & 298 & 1 & 152.903 & 298 & 25.64 & $\mathrm{KICCOO}$ & WEMWEQ \\
\hline 751.879 & 298 & 3 & 674.729 & 298 & 25.72 & CAMTOA01 & GUCJAP \\
\hline 245.987 & 298 & 1 & 219.891 & 298 & 26.10 & BANAPQ10 & BANAQP10 \\
\hline 255.171 & 298 & 1 & 228.927 & 298 & 26.24 & KOJGUL & GOWZEX \\
\hline 404.124 & 298 & 1 & 377.771 & 298 & 26.35 & LAKTUM & GULTIQ \\
\hline 339.125 & 298 & 2 & 286.272 & 298 & 26.43 & FEDBUL & FEDBOF \\
\hline 222.968 & 173 & 1 & 200.345 & 298 & 26.72 & THEOPH01 & BAPLOT01 \\
\hline 240.051 & 173 & 2 & 190.916 & 298 & 26.77 & MAHDOP & LACJEE \\
\hline 433.323 & 298 & 1 & 406.533 & 298 & 26.79 & GOWCAW & GOWBOJ \\
\hline 943.713 & 100 & 7 & 780.041 & 298 & 27.31 & DAFNOO & RIZGEM \\
\hline 162.061 & 298 & 1 & 134.719 & 298 & 27.34 & IJEQET & LASPRT \\
\hline 556.954 & 123 & 8 & 342.306 & 100 & 27.38 & REGREA & HOXOCDO2 \\
\hline 318.952 & 123 & 7 & 132.389 & 220 & 27.61 & LEBKEI & McGregor, $2006^{2}$ \\
\hline 387.837 & 298 & 1 & 360.089 & 298 & 27.75 & LACTOSO3 & EYOCUQ01 \\
\hline 189.609 & 298 & 1 & 161.815 & 298 & 27.79 & LEZJEF & LEZJAB01 \\
\hline 387.266 & 298 & 1 & 352.736 & 170 & 27.89 & VALMOL & BERYAZ \\
\hline 320.919 & 298 & 1 & 292.974 & 298 & 27.95 & IKUROV & IKURUB \\
\hline 298.771 & 298 & 4 & 186.042 & 298 & 28.18 & BEWYAE & IYAXAH \\
\hline
\end{tabular}




\begin{tabular}{|c|c|c|c|c|c|c|c|}
\hline 261.105 & 105 & 2 & 212.141 & 298 & 28.19 & SEFSOL & VAYJIO \\
\hline 246.944 & 298 & 1 & 218.736 & 298 & 28.21 & RUWKEZ & RUWKAV \\
\hline 301.241 & 298 & 1 & 272.968 & 298 & 28.27 & DUHKEW & BURLOP \\
\hline 519.628 & 298 & 1 & 491.177 & 298 & 28.45 & SATSEL & DURDAV \\
\hline 449.947 & 298 & 3 & 364.543 & 298 & 28.47 & SUGBIF & YADSOL \\
\hline 420.430 & 298 & 0.25 & 413.302 & 298 & 28.51 & ZOYMUV & ZOYMOP \\
\hline 318.514 & 298 & 1.5 & 275.652 & 298 & 28.57 & THIRDN10 & BELZEX \\
\hline 706.274 & 298 & 4 & 591.965 & 298 & 28.58 & SUMMOC & SUMMIW \\
\hline 337.404 & 298 & 1 & 308.788 & 298 & 28.62 & ZOZVUF & WISGEK \\
\hline 427.411 & 298 & 2 & 369.187 & 298 & 29.11 & CIZQAD01 & MENMIB01 \\
\hline 138.149 & 193 & 1 & 108.812 & 173 & 29.47 & DIPMUK & NILYAI \\
\hline 415.591 & 173 & 2 & 357.679 & 173 & 29.49 & SUPKET & SUPKIX \\
\hline 234.637 & 298 & 1 & 205.109 & 298 & 29.53 & RUVPUT & RUVPON \\
\hline 238.085 & 298 & 0.5 & 223.236 & 298 & 29.70 & RAVZOD & RAVZIX \\
\hline 189.445 & 298 & 1 & 165.587 & 543 & 29.82 & YEJNEG & QAMXUY \\
\hline 425.088 & 298 & 1 & 395.229 & 298 & 29.86 & VUXGAW & DORMOM \\
\hline 148.110 & 298 & 1 & 117.846 & 298 & 30.26 & JIYWET & JOWWIB \\
\hline 245.713 & 143 & 1 & 217.034 & 173 & 30.29 & POSTAS & POSTEW01 \\
\hline 122.693 & 210 & 1 & 91.067 & 83 & 30.33 & BULMAW10 & TFACET \\
\hline 469.652 & 198 & 2.5 & 400.218 & 298 & 30.54 & AQOMEY & UNOGIN \\
\hline 259.588 & 298 & 1 & 229.026 & 298 & 30.56 & ZZZAMSO4 & OPENAN \\
\hline 256.298 & 153 & 1 & 226.293 & 153 & 30.64 & QIMKOM & QIMKIG \\
\hline 632.803 & 298 & 2 & 570.917 & 298 & 30.94 & ETUTEC & ZZZEIIO1 \\
\hline 313.850 & 298 & 1 & 274.159 & 90 & 31.31 & RAWBIA & RAWBEW01 \\
\hline 270.767 & 298 & 1 & 239.415 & 298 & 31.35 & HAXBUD & LABJON \\
\hline 322.807 & 298 & 1 & 291.106 & 298 & 31.70 & NOJWAK & NOJVUD \\
\hline 367.405 & 298 & 4 & 237.412 & 213 & 31.76 & EACLTH10 & HIVROT \\
\hline 229.703 & 298 & 1 & 197.869 & 298 & 31.83 & PUVMAU & SAQJEZ \\
\hline 306.633 & 298 & 0.33 & 296.123 & 298 & 31.85 & ANSFON01 & DAPSUO05 \\
\hline 531.019 & 298 & 1 & 499.130 & 298 & 31.89 & TOHGIG & OMIXUD \\
\hline 721.941 & 123 & 2 & 659.133 & 124 & 32.26 & HAKDUT & HAKDON \\
\hline 191.794 & 298 & 1 & 159.302 & 298 & 32.49 & SAJRUQ & SAJQUP \\
\hline 342.780 & 298 & 2 & 277.440 & 298 & 32.67 & AZTHPN & CIPWUT \\
\hline 422.043 & 298 & 1 & 389.372 & 298 & 32.67 & GAFVIS & VUXBAR \\
\hline 187.516 & 298 & 1 & 153.335 & 298 & 34.18 & CADVUY & YUYMOU \\
\hline 268.428 & 298 & 1 & 227.446 & 130 & 35.36 & SOWSIG & QATWUD \\
\hline 378.339 & 298 & 1 & 337.026 & 193 & 36.11 & IVUQIZ & IVUQOF \\
\hline 476.836 & 298 & 1 & 440.523 & 298 & 36.31 & AMEVET & AMEVAP \\
\hline 221.002 & 100 & 0.67 & 202.775 & 298 & 36.81 & HIPKAS & GLUCIT03 \\
\hline 508.288 & 298 & 1 & 471.403 & 298 & 36.89 & CAZWAB & CAZWEF \\
\hline 261.205 & 150 & 3 & 155.470 & 298 & 37.14 & XOMWOL & HXACAN12 \\
\hline 461.522 & 298 & 1 & 424.128 & 298 & 37.39 & QIQLUX & QIQLIL \\
\hline 218.083 & 298 & 0.5 & 199.367 & 298 & 37.43 & RATBOD & RATBIX \\
\hline 578.184 & 298 & 1 & 540.728 & 298 & 37.46 & GESKUK & GESKOE \\
\hline
\end{tabular}




\begin{tabular}{|l|l|c|c|c|c|l|l|}
\hline 253.092 & 100 & 1 & 222.702 & 298 & 37.76 & EDOKOX & RIZFEL \\
\hline 387.886 & 298 & 1 & 349.488 & 298 & 38.40 & THIMCH10 & GEYXOX \\
\hline 323.077 & 200 & 1 & 289.244 & 298 & 38.49 & WUTVIQ & RENCUI \\
\hline 343.921 & 298 & 1 & 305.122 & 298 & 38.80 & CAKFAV & BUBTIB \\
\hline 335.531 & 298 & 0.5 & 316.092 & 298 & 38.88 & UKORUH & IZAJUO \\
\hline 570.405 & 298 & 1 & 531.488 & 298 & 38.92 & FIFFUV & ACPRET \\
\hline 234.370 & 298 & 0.5 & 214.315 & 298 & 40.11 & AHEREK & JAYPUU \\
\hline 198.119 & 150 & 1 & 160.550 & 298 & 41.88 & YAKWAJ & BISMEVO4 \\
\hline 411.060 & 298 & 0.5 & 388.957 & 298 & 44.21 & VIPCUS & ZOKSUN \\
\hline 667.044 & 298 & 1 & 622.342 & 298 & 44.70 & REQWEP & REQWAL \\
\hline 406.115 & 298 & 1 & 361.070 & 298 & 45.05 & NINSIM & NINSEI \\
\hline 210.857 & 143 & 0.25 & 199.046 & 143 & 48.32 & EDUHIU & EDUGOZ \\
\hline 588.339 & 298 & 2 & 477.703 & 298 & 55.32 & DOFTAT & DOFSUM \\
\hline 285.821 & 253 & 0.25 & 269.329 & 253 & 66.40 & NABWET & NABWAP \\
\hline
\end{tabular}




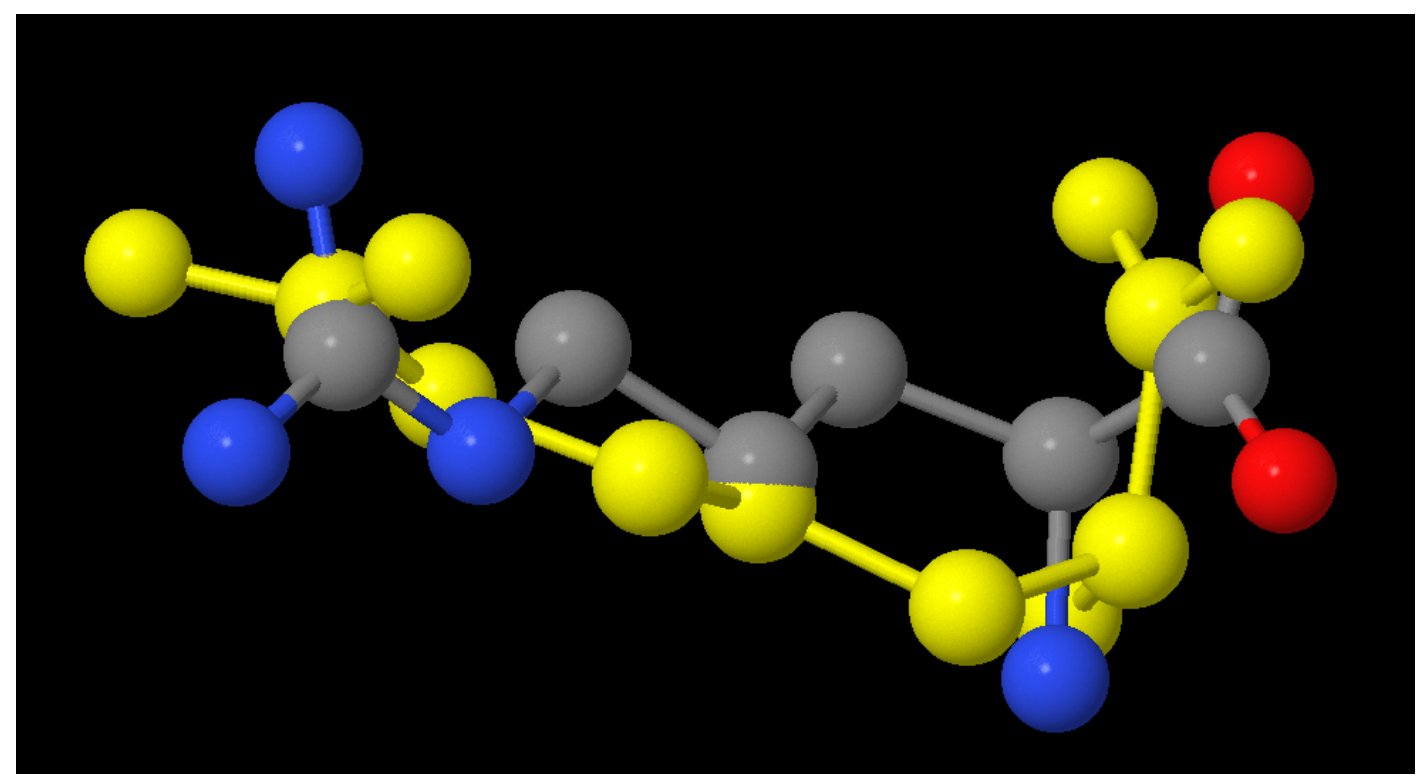

Figure S1: Overlay of molecular units from the amino-acid CSD files L-arginine L-

dihydrate, ${ }^{3}$ ARGIND.cif, and L-arginine, ${ }^{4}$ TAQBIY.cif $\left(\mathrm{C}_{6} \mathrm{H}_{14} \mathrm{~N}_{4} \mathrm{O}_{2}\right)$, illustrating the conformational changes between the hydrate and anhydrate in this flexible molecular system. One molecule has been rotated and translated as a rigid body to a minimised RMSD of $1.5 \AA$ with reference to the other molecule. This diagram has been prepared in Jmol using the “compare" function. ${ }^{5}$

\section{References}

1. van de Streek, J.; Motherwell, S., New software for searching the Cambridge Structural Database for solvated and unsolvated crystal structures applied to hydrates. CrystEngComm 2007, 9, 55-64.

2. McGregor, P. A.; Allan, D. R.; Parsons, S.; Clark, S. J., Hexamer formation in tertiary butyl alcohol (2-methyl-2-propanol, $\mathrm{C}_{4} \mathrm{H}_{10} \mathrm{O}$ ). Acta Crystallographica Section B Structural Science 2006, 62, 599-605.

3. Karle, I. L.; Karle, J., An application of the symbolic addition method to the structure of Larginine dihydrate. Acta Cryst. 1964, 17, 835-841.

4. $\quad$ Courvoisier, E.; Williams, P. A.; Lim, G. K.; Hughes, C. E.; Harris, K. D. M., The crystal structure of L-arginine. Chem. Comm. 2012, 48, 2761-2763.

5. Hanson, R. M., Jmol SMILES and Jmol SMARTS: specifications and applications. Journal of Cheminformatics 2016, 8, 50. 